\title{
BMJ Open Translation and cultural adaptation of the web and printed versions of a decision aid to support men's prostate cancer screening choice: a protocol
}

\author{
Sofia Baptista (1) , ${ }^{1,2}$ Bruno Heleno, ${ }^{3}$ Marta Pinto, ${ }^{2,4}$ Andreia Teixeira, ${ }^{1,2}$ \\ Kathryn L Taylor, ${ }^{5}$ Carlos Martins (1) ${ }^{1,2}$
}

To cite: Baptista S, Heleno B, Pinto M, et al. Translation and cultural adaptation of the web and printed versions of a decision aid to support men's prostate cancer screening choice: a protocol. BMJ Open 2019;9:e028938. doi:10.1136/ bmjopen-2019-028938

- Prepublication history for this paper is available online. To view these files, please visit the journal online (http://dx.doi. org/10.1136/bmjopen-2019028938).

Received 03 January 2019 Revised 29 March 2019 Accepted 18 June 2019

Check for updates

(c) Author(s) (or their employer(s)) 2019. Re-use permitted under CC BY-NC. No commercial re-use. See rights and permissions. Published by BMJ.

For numbered affiliations see end of article.

Correspondence to

Dr Sofia Baptista;

sofiatbaptista@gmail.com

\section{ABSTRACT}

Introduction Prostate cancer is one of the most frequent cancers among men. However, screening for prostate cancer carries many risks and a small benefit. Thus, based on the available evidence, most medical organisations advocate a shared decision-making process, in which decision aids may play an important role. Nevertheless, to date there is no such instrument to be used by Portuguese men. Our goal is to translate and perform the cultural adaptation of an English language prostate cancer screening decision aid called 'Making the best choice', in web and printed formats, which has been developed and tested by a workgroup from Georgetown University (USA). Methods and analysis Culturally and technically inappropriate recommendations in the original decision aid will be reviewed by the process coordinator and a linguistic expert. Two forward translations from English to Portuguese will be done, followed by a back-translation and an independent expert review. We will further improve the decision aid through an iterative process of data collection, data analysis and decision aid review. Individual semistructured cognitive interviews will be conducted and audiotaped with 55-69-year-old men recruited from the local community (urban and suburban) of Oporto district. We plan a total sample size of 30 participants (15 interviews per format). All participants will receive written information about the study and will sign individual consent forms. After verbatim transcription of the audiofiles, a thematic categorical analysis will be conducted using Ligre Software.

Ethics and dissemination The study protocol was approved by the Health Ethics Committee from Centro Hospitalar de São João/Faculdade de Medicina da Universidade do Porto. Results from this study will be disseminated in peer-reviewed publications and the Portuguese decision aid will available to Portuguese men.

\section{INTRODUCTION}

\section{Prostate cancer and screening}

Prostate cancer is the most common cancer among men worldwide ( 1.6 million cases) and the seventh leading cause of male cancer death, with a total of 366000 deaths. ${ }^{1}$ In Portugal, there are 90.5 new prostate cancer cases yearly per 100000 inhabitants, making
Strengths and limitations of this study

- This study will allow the development of the first Portuguese language decision aid to support Portuguese men's decision concerning prostate cancer screening.

- The major strength of this study is its purpose not solely to translate a previously tested English language decision aid but also to culturally adapt it.

- Another strength is the comprehension testing of the translated decision aid, using an iterative methodology consisting of individual cognitive interviews conducted with men aged 55-69 years recruited from the local community, which results will be incorporated to refine the final version of the Portuguese language decision aid.

- We will not measure our participants' health literacy or computer skills, which may limit our ability to assess appropriate audience for the intervention.

it the leading male cancer in terms of incidence. The adjusted mortality rate in 2014 was 20.3/100 000 with an absolute number of 1787 deaths from prostate cancer. In 2016, prostate cancer accounted for $1.7 \%$ of all deaths. $^{2}$

Screening can be defined as the use of simple tests across a healthy population to identify those individuals who have a disease, but do not yet have symptoms. ${ }^{3}$ Early detection refers to applying tests to asymptomatic individuals who consult a health professional. Throughout the text the term 'screening' will be used to refer both to systematic screening and early detection.

Screening for prostate cancer using a PSA (prostate specific antigen) test remains a controversial issue, implying a trade-off between benefits (low mortality reduction, possibility of an early diagnosis) and risks (high overdiagnosis and overtreatment with the consequent side effects, as well as false positive and false negative test results). ${ }^{4-7}$ 
Therefore, many guidelines issued by medical organisations such as the European Association of Urology, ${ }^{8}$ the American Cancer society, ${ }^{9}$ the United States Preventive Services Task Force, ${ }^{10}$ the American College of Physicians ${ }^{11}$ support a shared decision-making process regarding screening for prostate cancer, in which decision aids may play an important role.

In Portugal, the Directorate-General for Health (DGS), which is a governmental regulatory body of the technical and scientific aspects of healthcare, issued a national clinical guideline stating that the determination of PSA should not be used for a population-based screening for prostate cancer. In the case of early detection for asymptomatic men, this Portuguese consensus document advises a discussion between physician and patient about the benefits and adverse effects, pointing out the low mortality reduction and the risks of overdiagnosis and overtreatment. ${ }^{12}$ In spite of the scientific debate, PSA early detection is popular in Portugal. A 2013 population-based cross-sectional study showed that $67.3 \%$ of the Portuguese adult men consider that PSA test should be performed. ${ }^{13}$

\section{Cultural adaptation of decision AIDS}

According to the International Patient Decision Aids Standards Collaboration (IPDAS), decision aids are evidence-based tools designed to help people participate in decision making about healthcare options with the aim of improving the quality of the decision. ${ }^{14}$ IPDAS has developed a quality criteria framework for a document to qualify as a patient decision aid. Decision aids may be implemented in different formats, including written (eg, pamphlet/booklet), multimedia (eg, video, DVD) or web-based.

The most recent systematic review and meta-analysis assessing the impact of decision aids for screening decisions concluded that decision aids can increase patient knowledge, make people feel clearer about their values, reduce decisional conflict and promote an active patient role in decision making. ${ }^{15}$ Another meta-analysis, comparing web-based decision aids with other formats and usual care to support prostate cancer screening decision, found that in comparison with usual care, web-based decision aids significantly increased knowledge, reduced decisional conflict and reduced the practitioner-controlled role in the decision-making process; web-based decision aids performed similarly to alternative formats (printed, video) for the assessed decision-quality outcomes. ${ }^{16}$

The majority of world's population-about six billion people-may not have access to high-quality health information because it is in English. This illustrates how language can pose a global scale barrier. ${ }^{17}$ Portuguese is the world's sixth most spoken language and the fifth most used in the web. ${ }^{18}$ However, to date, there is no decision aid to support Portuguese men's decision regarding prostate cancer screening.

Nevertheless, translation alone is not enough. End-user utility is key; thus, delivery of quality care also depends on the accurate assessment and deeper understanding of the target's population cultural background. ${ }^{19}{ }^{20}$ Developing new decision aids can be time-consuming and costly. ${ }^{21}$ Consequently, adapting existing decision aids rather than developing new ones allows to benefit from previous fieldwork while avoiding duplication of developmental efforts and producing many similar decision aids. ${ }^{22}$

Although there is a growing body of literature on adapting and validating scales and health questionnaires, ${ }^{202324}$ decision aids are different tools, not intended to measure or assess but rather to foster patient involvement in a health decision, thus requiring a tailored adaptation methodology. However, little guidance is available concerning the process of cultural adaptation of existing decision aids. ${ }^{22}$

\section{Objectives}

We aim to make the translation and cultural adaptation of both printed and web formats of an English language patient decision aid addressing prostate cancer screening to be used by Portuguese men.

\section{METHODS AND ANALYSIS}

\section{Conceptual framework for adaptation}

We decided to follow the European Centre for Disease Prevention and Control's (ECDC) five-step, stakeholder-based approach to adapting health communication materials ${ }^{19}$ This approach was developed, tested and evaluated through a series of ECDC projects with public health practitioners, agencies and associations in seven European Union countries. ${ }^{25}$ It involves active engagement of patients, clinicians and researchers to ensure quality, comprehension, contextual and cultural appropriateness and applicability of any the adapted decision aids

\section{Step 1: Selection of materials and process coordinators}

We searched for prostate cancer screening decision aids in the A to Z inventory from the Ottawa Hospital Research Institute (https://decisionaid.ohri.ca/). The first author critically appraised the decision aids using IPDAS criteria. Thereafter, we identified those which fulfilled most of the quality criteria. Of those, we selected the 'Making the Best Choice' decision aid because it was presented in two different formats (paper and web). 'Making the best choice' is an English language prostate cancer screening decision aid which has been rigorously developed ${ }^{26}$ and extensively tested ${ }^{27-31}$ by a workgroup from Georgetown University (USA). We sought permission to translate and adapt the decision aid to the Portuguese population. KT, who was the principal investigator of the original decision aid, is a coinvestigator in this project.

Briefly, the printed and web versions of the decision aid share identical content and have an eighth-grade reading level. The informational sections include introductory material about the prostate gland; a description of screening tests and possible results; information about 
treatment options, risks and adverse effects; a review of prostate cancer risk factors and encouragement to discuss screening with a physician (but without instructions to make an immediate appointment); a 10-item values clarification tool; and resources for more information (references and a glossary). Additionally, the web decision aid includes pop-up definitions of 77 terms, video testimonials, an interactive values clarification tool and figures and animations. The decision aid has been found to improve prostate cancer knowledge and to reduce decisional conflict, with these changes remaining significant at the 13 months follow-up. Satisfaction was also higher for both formats in comparison with usual care; screening rates did not differ significantly among groups. ${ }^{28}$

We have reviewed the original printed and web versions of the decision aid and associated published data in close cooperation with its developers to identify the core elements of the decision aid namely, those concerning format and design features, structure, rationale and contents. SB is the process coordinator.

\section{Step 2: Early review}

Before starting the translation, the decision aid will be reviewed by the process coordinator and also by a linguistic expert. This early review aims to ensure that culturally and technically inappropriate recommendations are removed. Relevant national data and materials will be incorporated in the decision aid. In addition, the linguistic expert, who will do the translation later, will review the document, in close contact with one of the authors of the original document, to create a list of difficult concepts and alternative ways to convey the information.

Examples of changes after this early review include: (1) replacing the photos in the original decision aid with photos of Portuguese men; (2) replacing references to the American Societies/Associations' recommendations (with exception to USPSFT) by the Portuguese Directorate-General for Health's recommendations; (3) replacing American epidemiological data by Portuguese epidemiological data; (4) in a testimony there is this sentence: 'That's why I signed up for a free prostate screening programme.', this needs adaptation since it does not apply in the Portuguese healthcare service reality.

In addition, several years have lapsed since the original decision aid was developed. Therefore, we will perform a rapid review of clinical practice guidelines and systematic reviews of randomised controlled trials, and we will update estimates of benefit and harm of prostate cancer early detection if needed.

\section{Step 3: Translation and back-translation}

Two forward translations from English to Portuguese will be made, one of which by the process coordinator (native in Portuguese and fluent in English) and the other one by a professional translator, native of Portuguese. A consensus translated version will be obtained after discussion between the translators and the research group. The decision aid will then be back-translated by a professional translator, native speaker of English, fluent in Portuguese. The back-translated version will be discussed with KT to identify problems in the translation process. After this, an independent expert, who also understands the source language, will review the decision aid.

\section{Step 4: Comprehension testing}

This step aims to ensure the adapted version of the decision aid still retains its equivalence in an applied situation with target end-users.

\section{Participants}

Eligible participants will be recruited from the local community through advertisements in Porto district in streets, shops, churches and social media between January and February 2019. Portuguese native-speaking men aged 55-69 years old with a minimum sixth grade reading level (assessed by self-report) will be considered eligible, regardless of having been previously screened with a PSA test or not. A previous diagnosis of prostate cancer will be exclusion criteria. Candidates who respond to the advertisement will be invited for individual semistructured interviews to take place in the Faculty of Medicine of Porto or another place of convenience for the participant, providing confidentiality is assured. All participants will receive written information about the study and will sign individual consent forms.

We plan a total sample size of 30 participants (15 semistructured interviews for the printed decision aid and another 15 for the web-based decision aid).

\section{Individual semistructured interviews}

The interviews will be conducted by three family medicine residents (who are not involved in participants' healthcare) in accordance with an interview guide. Following participants consent to participate in the interview and to have it audiorecorded, they will be presented a general description of the decision aid and the study aims and will be asked to fill a questionnaire with basic demographic data. During the individual interviews, researchers will apply cognitive interview methods, using think aloud, probing and paraphrasing techniques ${ }^{32}$. Each participant will be provided the printed decision aid or a computer with access to the web-based format of the decision aid. Men will be asked to share their impressions aloud while they are going through the decision aid with the purpose of identifying potential issues in the format and content. ${ }^{22}$ Participants will also be encouraged to rephrase in their own words some sentences they may find more difficult to understand. In addition, at the end of the interview, a probing technique will be used retrospectively with questions to elicit how men felt throughout the process.

We plan to do 10 interviews, analyse them and update the decision aid according to the interviewees' feedback. Thereafter, we plan to do a last round of 5 interviews with the corrected decision aid in order to further refine it 
and in accordance with the desired iterative approach at the core of qualitative research.

\section{Data analysis}

Participants' demographic data will be analysed. Each interview will be given an alphanumeric code to omit participant's identity and thus ensure confidentiality. After verbatim transcription of the audiofiles, data obtained during the individual semistructured interviews will be analysed in a personal computer belonging to one of the authors, ensuring it has not a network connection. The audiofiles will be destroyed, at maximum, 1 month after data analysis. A thematic categorical analysis will be conducted using Ligre Software. Two authors will independently fill a content analysis form and the concordance level will be assessed. We will cluster similar ideas together in order to categorise issues with (1) navigation and usability of the interface, (2) content comprehension and completeness, (3) sociocultural appropriateness, (4) length of decision aid and amount of information, (5) use of figures/illustrations, (6) time of completion. ${ }^{21}$ Atypical cases that do not fit patterns identified for the majority will also be identified. In addition, we will analyse specific problems, as well as the participants' proposed solutions or revisions.

\section{Step 5: Proofreading}

Proofreading will be conducted by two Portuguese native speakers selected by the process coordinator, who have not read the decision aid before. There will be two proofreading moments, the second of which will be done in the final format of the printed decision aid and website, respectively, with special attention to the design features.

\section{Public involvement statement}

As stated previously in this section, we intend to involve the public who will be the end target user of the decision aid in a crucial point of the research process: the field test of the decision aid. During that phase, the qualitative methodology will include individual cognitive interviews. The methods were designed to get the most out of participants' perceptions, experience and opinions and to use them to directly improve and ultimately refine the final version of the decision aid. In addition, public will also be the core part of our dissemination plan, as we aim to create the first Portuguese decision aid to support Portuguese men's prostate cancer screening decision, both in web and printed formats.

\section{ETHICS AND DISSEMINATION}

Results from this study will be disseminated in peer-reviewed publications, conference presentations, reports and in a PhD thesis. The translated and culturally adapted decision aid is intended to be delivered to Portuguese men to support their decision regarding prostate cancer screening.
Author affiliations

${ }^{1}$ Department of Community Medicine, Information and Health Decision Sciences (MEDCIDS), University of Porto Faculty of Medicine, Porto, Portugal

${ }^{2}$ Centre for Health Technology and Services Research (CINTESIS), University of Porto, Porto, Portugal

${ }^{3}$ CEDOC, Chronic Diseases Research Centre, NOVA Medical School, Lisboa, Portugal ${ }^{4}$ Psychology of Deviance and Justice, Faculty of Psychology and Education Sciences, University of Porto, Porto, Portugal

${ }^{5}$ Department of Oncology, Lombardi Comprehensive Cancer Center, Georgetown University, Washington, Columbia, USA

Contributors $\mathrm{SB}, \mathrm{AT}, \mathrm{MP}, \mathrm{BH}, \mathrm{KLT}$ and $\mathrm{CM}$ were involved in the design of the protocol for the study. SB wrote the first draft of this manuscript. AT, BH, MP, KLT and $\mathrm{CM}$ critically contributed to and refined this manuscript. All authors approved the final manuscript.

Funding This article was supported by National Funds through FCT-Fundação para a Ciência e a Tecnologia within CINTESIS, R\&D Unit (UID/IC/4255/2019).

Competing interests None declared.

Patient consent for publication Not required.

Ethics approval The study protocol was approved by the Health Ethics Committee from Centro Hospitalar de São João/Faculdade de Medicina da Universidade do Porto ID.: 339/18

Provenance and peer review Not commissioned; externally peer reviewed.

Open access This is an open access article distributed in accordance with the Creative Commons Attribution Non Commercial (CC BY-NC 4.0) license, which permits others to distribute, remix, adapt, build upon this work non-commercially, and license their derivative works on different terms, provided the original work is properly cited, appropriate credit is given, any changes made indicated, and the use is non-commercial. See: http://creativecommons.org/licenses/by-nc/4.0/.

\section{ORCID iDs}

Sofia Baptista http://orcid.org/0000-0002-2604-9369

Carlos Martins http://orcid.org/0000-0001-8561-5167

\section{REFERENCES}

1. Fitzmaurice C, Allen C, Barber RM, et al. Global, regional, and National cancer incidence, mortality, years of life lost, years lived with disability, and Disability-Adjusted life-years for 32 cancer groups, 1990 to 2015: a systematic analysis for the global burden of disease study. JAMA Oncol 2017;3:524.

2. Direcção Geral de Saúde. Programa Nacional para as Doenças Oncológicas. Portugal: Doenças Oncológicas em Números, 2015.

3. WHO. Early detection of cancer. Available: https://www.who.int/ cancer/detection/en/ [Accessed Mar 2019].

4. Andriole GL, Crawford ED, Grubb RL, et al. Prostate cancer screening in the randomized prostate, lung, colorectal, and ovarian cancer screening trial: mortality results after 13 years of follow-up. $J$ Natl Cancer Inst 2012;104:125-32.

5. Schröder FH, Hugosson J, Roobol MJ, et al. Screening and prostate cancer mortality: results of the European randomised study of screening for prostate cancer (ERSPC) at 13 years of follow-up. Lancet 2014;384:2027-35.

6. Heijnsdijk EAM, Wever EM, Auvinen A, et al. Quality-Of-Life effects of prostate-specific antigen screening. N Engl J Med 2012;367:595-605.

7. Martin RM, Donovan JL, Turner EL, et al. Effect of a low-intensity PSA-Based screening intervention on prostate cancer mortality: the cap randomized clinical trial. JAMA 2018;319:883-95.

8. Mottet N, Bellmunt J, Briers E, et al. EAU guidelines on prostate cancer, 2017. Available: http://uroweb.org/individual-guidelines/ oncology-guidelines/ [Accessed Dec 2018].

9. Wolf AMD, Wender RC, Etzioni RB, et al. American cancer Society guideline for the early detection of prostate cancer: update 2010. CA Cancer J Clin 2010;60:70-98.

10. Final Recommendation Statement. Screening for prostate cancer and final evidence review: screening for prostate cancer. U.S. Preventive Services Task Force, 2018.

11. Qaseem A, Barry MJ, Denberg TD, et al. Screening for prostate cancer: a guidance statement from the clinical guidelines Committee of the American College of physicians. Ann Intern Med 2013;158:761. 
12. Saúde DGde. Prescrição E Determinação do Antigénio Especifico dA Próstata - PSA. Norma de Orientação Clínica 060/2011, 2017.

13. Martins C, Azevedo LF, Ribeiro O, et al. A population-based nationwide cross-sectional study on preventive health services utilization in Portugal-What services (and frequencies) are deemed necessary by patients? PLoS One 2013;8:e81256.

14. Volk R, H L-T. Update of the International Patient Decision Aids Standards (IPDAS) Collaboration's background document, 2012. http://ipdas.ohri.ca/IPDAS-Introduction.pdf

15. Stacey D, Légaré F, Lewis $\mathrm{K}$, et al. Decision AIDS for people facing health treatment or screening decisions. Cochrane Database Syst Rev 2017;19.

16. Baptista S, Teles Sampaio E, Heleno B, et al. Web-Based versus usual care and other formats of decision AIDS to support prostate cancer screening decisions: systematic review and meta-analysis. $J$ Med Internet Res 2018;20:e228.

17. Adams P, Fleck F. Bridging the language divide in health. Bull World Health Organ 2015;93:365-6.

18. Internet World Stats. Internet world users by language. Available: https://www.internetworldstats.com/stats7.htm [Accessed Dec 2018].

19. European Centre for Disease Prevention and Control. Translation is not enough - cultural adaptation of health communication materials. Stockholm: ECDC, 2016.

20. Sousa VD, Rojjanasrirat W, Translation RW. Translation, adaptation and validation of instruments or scales for use in cross-cultural health care research: a clear and user-friendly guideline. J Eval Clin Pract 2011;17:268-74.

21. Coulter A, Stilwell D, Kryworuchko J, et al. A systematic development process for patient decision AIDS. BMC Med Inform Decis Mak 2013;13:S2.
22. Chenel V, Mortenson W, Guay M, et al. Cultural adaptation and validation of patient decision AIDS: a scoping review. Patient Prefer Adherence 2018;12:321-32.

23. Beaton DE, Bombardier C, Guillemin F, et al. Guidelines for the process of cross-cultural adaptation of self-report measures. Spine 2000;25:3186-91.

24. Kurre A, van Gool CJAW, Bastiaenen CHG, et al. Translation, cross-cultural adaptation and reliability of the German version of the dizziness handicap inventory. Otology \& Neurotology 2009;30:359-67.

25. European Centre for Disease Prevention and Control. Let's talk about protection. Stockholm: ECDC, 2012.

26. Dorfman CS, Williams RM, Kassan EC, et al. The development of a web- and a print-based decision aid for prostate cancer screening. BMC Med Inform Decis Mak 2010;10:12.

27. Kassan EC, Williams RM, Kelly SP, et al. Men's use of an Internetbased decision aid for prostate cancer screening. $J$ Health Commun 2012;17:677-97.

28. Taylor KL, Williams RM, Davis K, et al. Decision making in prostate cancer screening using decision AIDS vs usual care: a randomized clinical trial. JAMA Intern Med 2013;173:1704-12.

29. Tomko C, Davis KM, Luta G, et al. A comparison of web-based versus print-based decision AIDS for prostate cancer screening: participants' evaluation and utilization. J Gen Intern Med 2015;30:33-42.

30. Tomko C, Davis K, Ludin S, et al. Decisional outcomes following use of an interactive web-based decision aid for prostate cancer screening. Transl Behav Med 2015;5:189-97.

31. Starosta AJ, Luta G, Tomko CA, et al. Baseline attitudes about prostate cancer screening moderate the impact of decision AIDS on screening rates. Ann Behav Med 2015;49:762-8.

32. Collins D. Pretesting survey instruments: an overview of cognitive methods. Qual Life Res 2003;12:229-38. 